\title{
Designs and Development of Engine Measurement System for Experiment Test
}

\author{
Zongzheng Ma, Shaohua Dong, Qianzhu Zhang, Bobo Pan, Yan Huang \\ Department of Mechanical Engineering, \\ Henan Institute of Engineering \\ Zhengzhou, China
}

\begin{abstract}
With the development of computer technology, data acquisition is more and more easily which can replace the manual recording process and improve the accuracy and efficiency of the data. So one experiment data acquisition system for the internal combustion (IC) engine was designed including the hardware and software system. And the hardware was completed based on the different sensor and sensor processing circuit, meanwhile, the software of the data acquisition system is realized based on $\mathrm{VC}++$ platform. Then the real experiment was done using this system and accurate data was achieved based on the engine. It is proved by the experiment test that the measurement system can achieve engine cylinder pressure measure. So this data acquisition system is helpful to improve the understanding of the theoretical knowledge.
\end{abstract}

Keywords—data acquisition; engine experiment; hardware; software; design

\section{INTRODUCTION}

With the development of computer technology in all areas, real-time data acquisition, data display, real-time control are possible for the experiment measurement which can reduce the manual recording and ensure accurate and effective data acquisition.

In the 50 s of 19 th century the data acquisition device began to appear. US-developed test system for data acquisition is the symbol which was mainly used in the military field. This kind of data acquisition device can be operated by person who is not needed to familiar with the system. At the late 1960s, data acquisition products appeared in foreign markets and these data acquisition products are used in more specific areas. At the late 1970s, with the development of micro-computer, data acquisition and control computer equipment acquisition integration appeared. Then in the 1980s, with the popularity of computer the universal data acquisition device began to appear and it is mainly composed by the acquisition board, interface bus communication based on GPIB and control computer [1]. But at the late 1980s, the hardware is replaced by the software which can reduce the cost and size of the data acquisition system and increase its performance. After the 1990s, the data acquisition technology has been widely used in military, aerospace and industrial applications in developed countries. With the development of integrated circuit technology, there has been a high-performance single-chip data acquisition system DAS whose resolution is 16 bit and the sampling rate is up to hundreds of thousands times per second. And at this period, data collection devices modular structure was divided into parallel bus and serial bus. However, due to the prices and some specialized systems, the universal data acquisition system is not suitable for all application [2]. Then one data acquisition system based on $\mathrm{VC}++$ platform is designed in this paper for the engine experiment measurement.

The hardware of the system is divided into data acquisition, real-time signal acquisition module and data processing module which can be seen in figure 1 .

The data acquisition includes the sensor and communication system. The real-time signal acquisition module is the PCI data acquisition card. And the data processing module is the computer software.

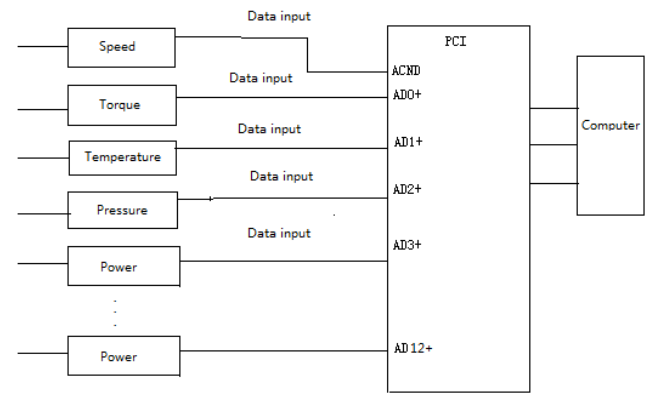

Fig. 1. Diagram of the system

\section{SENSOR PROCESSING}

The sensor signal can be divided into analog and digital signal. The digital signal can be input the computer directly but the analog signal should be processed. For the IC engine, most of the sensor signals are analog signal which can be roughly classified into linear signal and the sine signal.

\section{A. Sine signal processing method}

In order to determine the dead center (DC) position of the engine the magnetic sensors was used in the engine. According to the output characteristics of the magnetic sensors the output analog signal is sine signal which is shown in Fig. 2.

Since the position between the top dead center (TDC) and the magnetic sensors is fixed, the magnetic sensors signal can be used to determine the TDC position and it also can be used to calculate the speed $[3,4]$. In addition, in order to be input into the computer the first thing is to convert the magnetic signals to TTL signal (digital signal). The 
processing circuit is shown in Fig. 3, where the 6N137 is used as digital switching which the sine signal is converted into digital signal (Fig. 4). Then the signal can be directly input microcontroller utilizing the $\mathrm{I} / \mathrm{O}$ port [5].

It should be noted that this method has error due to that there is transmission time for the 6 N137 chip.

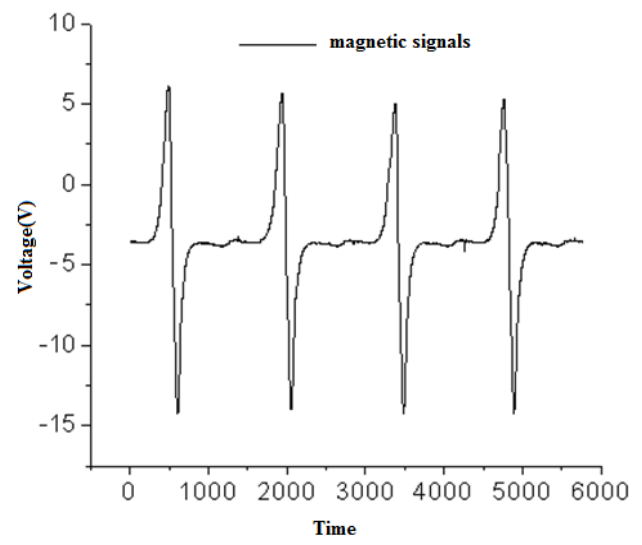

Fig. 2. The output signal of magnetic sensor

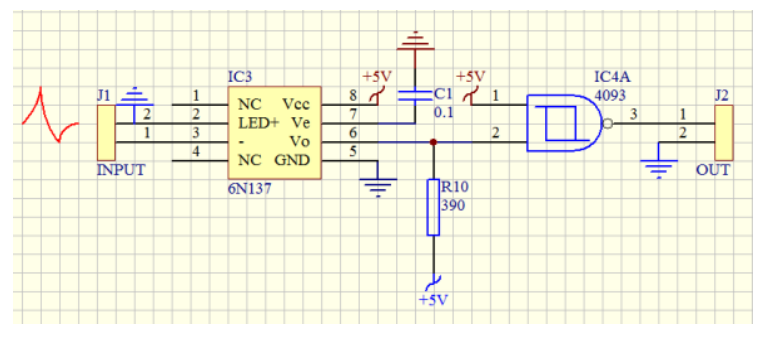

Fig. 3. Diagram of processing circuit

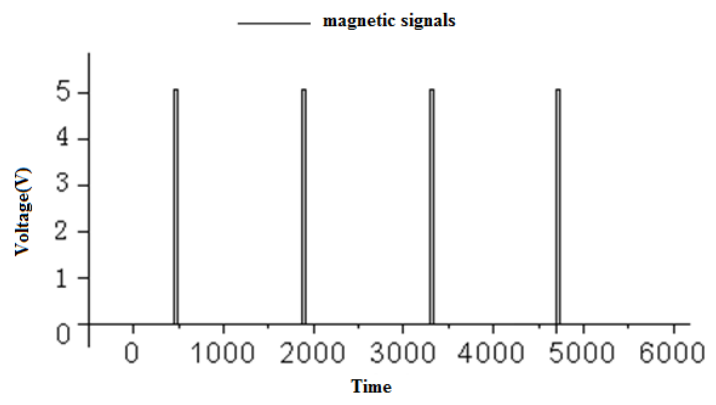

Fig. 4. The convert signal

\section{B. Linear signal processing method}

If the output of the analog signal is voltage value and there is a linear relationship between the measured parameter and the output signal, it can be input the computer by $A / D$ converter [6]. However, if the amplitude of the analog signal is small the A/D conversion precision cannot be full used and the signal should be enlarged while the limit processing is required if the amplitude is large. For the
IC engine, the outputs signals of the throttle position sensor signal and the intake pressure sensor are linear which are shown in Fig. 5. And the output signal is a voltage signal between 0 5V whose amplitude range is within the PCI data acquisition capacity and it can be input directly to $A / D$ ports.

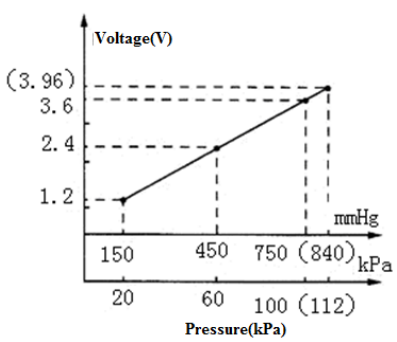

(a) Intake air pressure

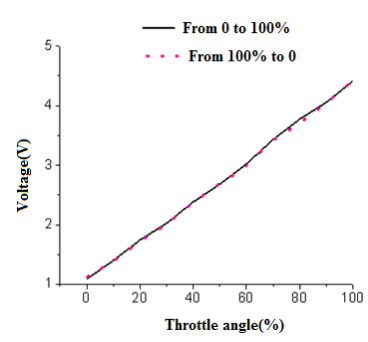

(b) Throttle vale angle
Fig. 5. Liner signals

\section{SOFTWARE DESIGN}

In this part, the programming process is emphasized based on $\mathrm{VC}++$ platform and it can be achieved through two stages, basic interface design and coding.

\section{A. Basic interface design}

The basic interface design was build based on basic controller including the text controller, edit controller, button controller and Com-box controller.

For text controller, its function is data and label display and it is used to display the input and output parameters.

For the edit controller, it can be used to input initial data which can realize the copy, cut, paste and delete operation. The button controller is also common used in basic interface design and its ability is to achieve calculation.

The Com-box controller combines the features of edit box and list box which can be used to data input and list box selection. In the basic interface the Com-box controller is used to display the curve.

The basic interface divided into three parts, the first part is data input, the second part is value monitor and the third part is data acquisition, which is shown in Fig. 6.

In the data input part, the basic parameters such as engine parameters, experiment time, experiment place, experiment temperature, path of storage can be input.

In the monitor part, the different channel value can be checked using the curve display.

In the data acquisition part, the value can be stored in the computer. 


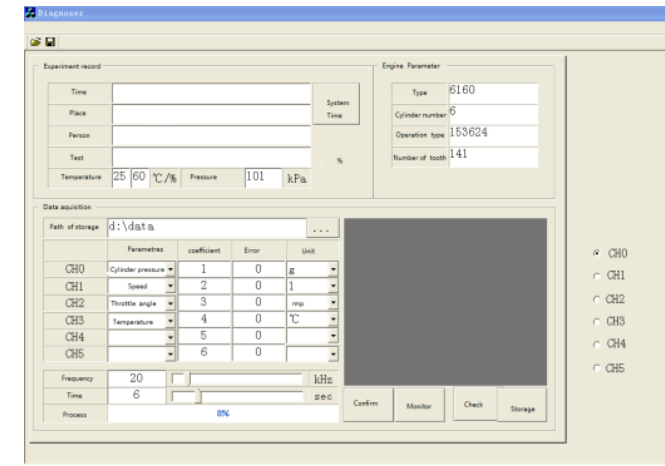

Fig. 6. Main interface of the system

\section{USING THE TEMPLATE}

\section{A. 3.2 Coding}

The main coding process includes the data acquisition and data storage which are designed based on $\mathrm{VC}++[7]$. And the data acquisition coding is shown in the following.

void CDiagnoserView::OnConfirm()

\{

UpdateData(true);

biCHselect $=0$;

bFirstCH=0;

for(short $\mathrm{i}=0 ; \mathrm{i}<6 ; \mathrm{i}++)$

\{

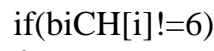

bFirstCH=i;

\}

biCHselect++;

\}

\section{FILE $* \mathrm{fp}$}

$\mathrm{fp}=$ fopen $(" \mathrm{~d}:$ IIExperiment.txt","w");

fprintf(fp, "Experiment time: \%s\n", m_strTime);

fprintf (fp, "Experiment place:\%s\n", m_strArea);

fprintf(fp,"Experiment person: \%s\n", m_str People);

fprintf(fp, "Experiment test:\%s\n", m_strWork);

fprintf(fp, "Temperature:\%d; Humidity: \%d; m_siTemperature, m_siHumidity, m_siPressure);

fprintf(fp,"Engine parameters: $\quad \mathrm{n}$ ");

fprintf(fp,"Type : \%s; Cylinder number : \%d; Operation type: \%s; Number of tooth: \%d\n",

m_strModal,m_siCylinder,m_strSequence,m_siGear); fclose(fp);

$\mathrm{fp}=$ fopen("c:॥para.ini","w"); fprintf(fp,"\%d \%d \%d \%d \%d

$\% d \backslash n ", m \_i C H 0 S e l e c t, m \_i C H 1 S e l e c t, m \_i C H 2 S e l e c t, m \_i C H$ 3Select,m_iCH4Select,m_iCH5Select); fprintf(fp,"\%f \%f \%f $\% \mathrm{f} \quad \% \mathrm{f}$ $\%$ f\n",m_fScale0,m_fScale1,m_fScale2,m_fScale3,m_fScal e4,m_fScale5); fprintf(fp,"\%f $\% \mathrm{f} \quad \% \mathrm{f} \quad \% \mathrm{f} \quad \% \mathrm{f}$ $\%$ fln",m_fOff0,m_fOff1,m_fOff2,m_fOff3,m_fOff $4, m \_f O f$ f5);

fprintf(fp,"\%d $\% d \quad \% d \quad \% d \quad \% d$ $\% d \backslash n ", m \_i U n i t 0, m \_i U n i t 1, m \_i U n i t 2, m \_i U n i t 3, m \_i U n i t 4, m$ _iUnit5);

fprintf(fp,"\%d \%dln",bFirstCH,biCHselect); for $(\mathrm{i}=0 ; \mathrm{i}<6 ; \mathrm{i}++)$

\{

\} fprintf(fp,"\%d ",biCH[i]); for $(\mathrm{i}=0 ; \mathrm{i}<6 ; \mathrm{i}++)$

\{ \} fprintf(fp,"\%s ",strChSet[i]); fclose(fp); \}

\section{APPLICATION}

The experiment test bed consists of K157 FMI engine (specific parameters shown in table 1), electric eddy current dynamometer, dynamometer control system, gasoline engine ECU, computer and data acquisition system and sensors of related parameters. The experiment test bed is shown in figure 7, where the electric eddy current dynamometer and dynamometer control system are used to control and measure the load of the engine, engine fuel injection system control fuel injection and ignition, and the computer's serial communication can also be used to control gasoline engine ECU as to control fuel injection and ignition.

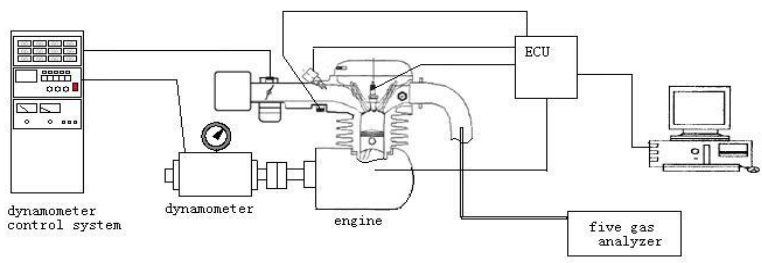

Fig. 7. Diagram of experimental system

TABLE I. K157 FMI ENGINE PARAMETERS

\begin{tabular}{|c|c|}
\hline Parameter & Value \\
\hline Type & $\begin{array}{c}\text { Single cylinder, } \\
\text { four-stroke }\end{array}$ \\
\hline Bore $\times$ stroke $(\mathrm{mm})$ & $56.5 \times 49.5$ \\
\hline Total displacement $/ \mathrm{L}$ & 124 \\
\hline Rated power $(\mathrm{kW}) /(\mathrm{r} / \mathrm{min})$ & $7.0 / 7500$ \\
\hline Compression ratio & $9: 1$ \\
\hline Cooling style & wind cooling \\
\hline
\end{tabular}

The cylinder pressure measured using the data acquisition system can be seen in figure 8 when the engine speed is $1500 \mathrm{r} / \mathrm{min}$ and throttle opening angle is $20 \%$ of wide open throttle. 
It can be seen from the figure that the highest cylinder is $1.05 \mathrm{MPa}$ which appears at $374^{\circ} \mathrm{CA}$ (Crank angle) under this speed. It can be concluded that this system can realize data acquisition and is helpful to the understanding of the theoretical knowledge.

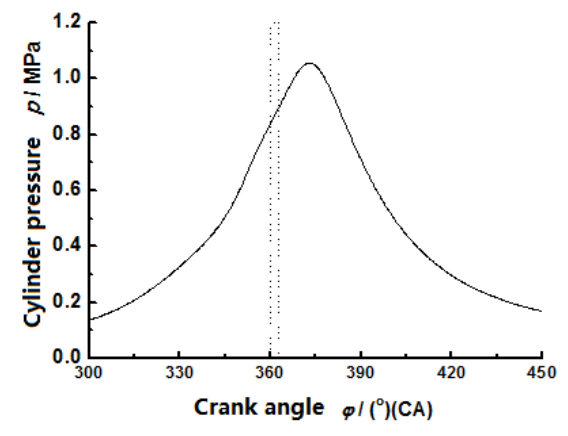

Fig. 8. Real cylinder pressure

\section{CONCLUSION}

In this paper, one data acquisition system for engine experiment is designed including the hardware and software. The design of the controller hardware system is completed firstly, and then the sensor processing is improved. Meanwhile, the coding of the system has been designed. At the end this system was validated by real cylinder pressure measure experiment.

\section{ACKNOWLEDGEMENT}

The authors would like to express appreciation of financial support by the Key Subject of Machine Design and Theory, the Reliability Test Center and Modern Design Method Research Center of Henan Institute of Engineering.

\section{REFERENCES}

[1] Y.P. Xu, Z.Y. Cao, and D.P. Xu, "The Development of Managemen and Acquisition Software in the High Speed Data Acquisition System, " 2nd International Symposium on Instrumentation Science and Technology, vol.1,pp. 367-369, August 2002.

[2] Y. B.Geng, and X. D. Zhang, "A Data Acquisition System Based on PCI Bus for the Digital Nuclear Magnetic Resonance Image Spectrometer," The Seventh International Conference on Electronic Measurement and Instruments, vol.6,pp. 89-91,August 2005.

[3] L. T. Ding L T, R. C. Li, and Q. Z. Wang, "Crankshaft crank shaft strength and fatigue strength of research methods to improve life," Tractor \& Farm Transporter, vol.6,pp. 18-20,December 2004.

[4] J. F. Kong, "1.3L gasoline engine crankshaft strength analysis of car," Shanghai: Shanghai Communications University, 2007.

[5] S. T. He, and Z. Y. He, "Mode Analysis of Three dimensional Finite Element and Experimental Study on a 6102B Diesel Engine Crankshaft," Transactions of The Chinese Society of Agricultural Machinery, vol32,pp.74-77,April 2001.

[6] Y. L. Wang, Mechanics of Materials. Beijing: Mechanical Industry Press, 2009.

[7] S. Kun, R. N. Li, Visual C ++ programming self-study manual. Beijing: People Post Press, 2008. 